\title{
D
}

${ }^{1}$ Center for interstitial and rare lung diseases, Pneumology and respiratory critical care medicine, Thoraxklinik, University of Heidelberg, Heidelberg, Germany. ${ }^{2}$ University Clinic of Pulmonology and Allergy, Skopje, Macedonia. ${ }^{3}$ Pulmonology, Critical Care and Sleep Medicine, B P Koirala Institute of Health Sciences, Dharan, Nepal.

\section{Interstitial lung diseases: course report}

\section{Course organisers}

M. Kreuter, F.J.F. Herth, T. Geiser

\section{Faculty}

J. Behr, V. Cottin, B. Crestani, E. Eber, R. Eberhardt, O. Eickelberg, C.P. Heussel, N. Kahn, T. Maher, P. Riddell, A.-M. Russell, M. Vasakova, A. Warth, W. Wuyts

\section{Introduction}

This course demonstrated how to diagnose interstitial lung diseases (ILDs) correctly, and highlighted the limitations and pitfalls of the different approaches. It examined the current standards of ILD treatment, its challenges and identified promising new developments within this topic area. Participants were able to discuss their own patient cases with an interdisciplinary ILD board. The course included case presentations with interactive discussions, plenary lectures and workshops.

The course took place on April 14-16, 2016 in Heidelberg, Germany, with 92 participants, the majority of whom were clinicians. Most attendees already had a high level of knowledge about ILDs, but were excited to acquire more knowledge in this field, especially concerning radiology and pathology. Participants were highly interested to gain insights into the most current updates on all forms of ILDs and especially to discuss their own cases with the faculty and their co-participants.
Participants were eager to engage themselves in the workshops to learn about the interpretation of high-resolution computed tomography (HRCT), gain some understanding of the histopathology of ILDs and to discuss current clinical problems in ILD. Moreover, there were lively discussions about the cases submitted by the faculty and by the participants, with a very high learning input for participants. The interactive sessions emerged as the best educational activities, but participants were also keen to get the most up-to-date information on different ILDs. Many participants asked for a repetition of this course, amended by the addition of more interactive sessions.

\section{Sead Zeynel, Macedonia, a participant}

Since there is a lack of proper study modules within the syllabuses of our specialisation this educational opportunity, for which I received an ERS bursary, has been of great benefit for me and my home institution. The ILDs, as a heterogeneous group of disorders, are associated with considerable morbidity and mortality in my country. Participation in this ERS Course enabled me to gain a clearer insight into the topic of ILDs, in which I'm particularly interested, lighting my clinical and scientific curiosity.

The programme was comprehensive, covering ILDs from a general overview of diagnosis to the specific diseases and their particularities in terms
Cite as: Kreuter M, Zeynel S, Mishra DR. Interstitial lung diseases: course report. Breathe 2016; 12: 213-215. 
of diagnosis and therapy. The programme conveyed its content through systematic and highly interactive presentations and workshop sessions, accompanied by quizzes, delivered by experts in the field and enthusiastic speakers. My aim was that the knowledge and skills acquired would enable me to approach patients with raised awareness of this general category of diseases, which includes many different lung conditions, and attending this course has given me guidance to help me achieve my objectives for the ultimate benefit of my patients.

Participation in this course has been a great opportunity to network with prominent experts in the field, as well as gain a general overview through an intense course that is much needed for proper orientation through the process of career development in this particular field of pulmonary medicine.

The predominant atmosphere was vivid, pleasant and comfortable. The programme was not overwhelming, maintaining an adequate tempo of sessions and breaks, enabling participants to stay focused throughout the programme. The course venue, Rohrbacher Schlösschen, had a positive impact on the above description of the predominant atmosphere, as much as the Thoraxklinik at Heidelberg University Hospital and the beautiful city of Heidelberg.

In my opinion, the most controversial issues among the participants concerned diagnostic accuracy and the question of whether we know what we are treating, which has to do with the peculiarity of the ILDs in general as well the specific diseases.

After this course I have a deeper insight into the ILDs and their subgroups. In terms of this, as participants, we have comprehensively covered idiopathic pulmonary fibrosis (IPF) and the phenomenon of "acute exacerbation". Moreover, we were provided with presentations increasing our awareness of the subgroup of ILDs of unknown aetiology that is the interstitial pneumonias, which are associated with variable radiological features, response to therapy and clinical course.

As respiratory physicians encountering patients that may be diagnosed with a certain ILD, the deeper understanding of the histological patterns and their disease associations contributes towards better multidisciplinary collaboration. We were able to see to what extent a respiratory physician and a pathologist are interdependent in the diagnosis of certain ILDs. Being knowledgeable of the different histological patterns, one can have in mind the patient's prognosis. For example, patients with nonspecific interstitial pneumonia, fare better than those with usual interstitial pneumonia (UIP).

Among the other knowledge that I have acquired during the course, I would mention the conventional chest radiographic assessment and classification of ILDs according to the pattern on chest radiographs (alveolar filling and interstitial

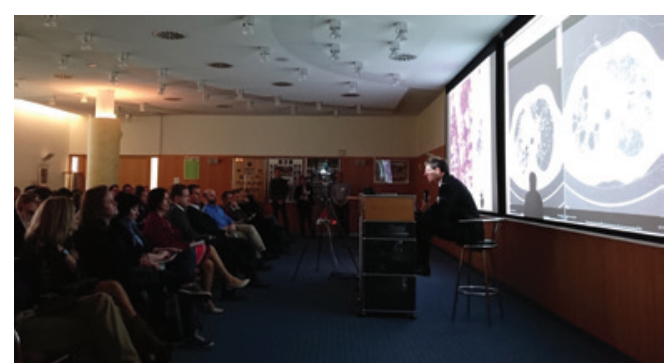

pattern, and mixed pattern). Moreover, I have gained invaluable knowledge and skills in HRCT interpretation for ILDs and different radiological patterns, along with practical skills in the different histology patterns, all of which were put together in the clinical context. In this regard, I have gained skills in recognising the HRCT patterns, i.e. UIP, possible UIP and a pattern inconsistent with UIP. The rotating practical workshops contributed a great deal to this.

Going through the different specific ILDs, which were enhanced by several multidisciplinary ILD board case discussions, I have become aware of the importance of raised suspicion for hypersensitivity pneumonitis, especially in cases of early disease recognition and exposure removal, as well as gaining an awareness of the poor prognosis of patients with chronic fibrotic or emphysematous manifestations. For the primary (unclassified) ILDS, e.g. sarcoidosis and connective tissue disease-associated ILDs, where the lung can be involved by a wide variety of pathologies, specific pathological entities that are seen in these diseases do not generally follow the same course as their idiopathic counterparts.

One of the parts of the programme of particular interest was the section on ILD comorbidities. In the lungs, parenchymal and vascular remodelling share pathomechanisms that may explain the relatively high prevalence (30-40\%) of pulmonary hypertension $(\mathrm{PH})$ in ILD patients. In the absence of satisfactory therapies for many ILDs, and considering the clinical burden of $\mathrm{PH}$ in affected patients, specific vasomodulatory therapies currently approved for pulmonary arterial hypertension may be promising options for ILD patients. Other presentations that incited interest were the lectures on cell therapy in ILDs, lung cancer in ILDs, and lung transplantation.

The future perspectives on ILDs, a session delivered at the end of the programme, was one of those that instilled determination to pursue a career devoted to ILDs as a diagnostic and treatment challenge for a respiratory physician.

\section{Deebya Raj Mishra, Nepal, a participant}

Being a pulmonology trainee from a developing country, Nepal, where pulmonology is, at best, 
in its nascent stages, the exposure to the exciting world of ILDs at Heidelberg was eye opening. For us, and maybe in other developing countries without access to newer advances, ILD has meant treatment with steroids and oxygen. The course taught me it is much more and helped bring me up-to-date with recent changes.

The course provided an in-depth look at the different facets of ILD. The topics covered ranged from the obvious IPF to relatively newer entities like combined pulmonary fibrosis with emphysema and pleuropulmonary fibroelastosis). The course was well distributed between lectures and workshops, and the focus on radiology and pathology aspects of ILD, more in tune with the multidisciplinary approach to diagnosis advocated nowadays, was especially impressive. It was interesting to realise the paradigm shift from an anti-inflammatory treatment approach to an anti-fibrotic treatment approach for IPF, an approach that is still not much practiced in developing countries. All the lectures also dealt with future directions and there was lot of optimism among all the speakers at the event. The focus on early preparation for lung transplantation (subject to availability and allocation) was well emphasised, along with encouragement of conversations about end of life strategies, a subject often ignored in resource-limited settings.

The course was held in the beautiful city of Heidelberg at the Thoraxklinik at Heidelberg University Hospital, a centre of excellence for respiratory diseases. The laidback atmosphere provided an opportunity for the participants to mingle, with guided city tours being provided by the organisers. The participants were an interesting mix, with the presence of a pathologist and a few radiologists as well as the pulmonologists. The participants included individuals from developing countries like Zimbabwe, Columbia and
Sri Lanka besides the majority that were based in European countries. It was a wonderful opportunity to network with fellow colleagues and seniors. The faculty were all distinguished and eminent leaders in different facets of ILDs, with some having expertise in rare and orphan lung diseases, while others were leading researchers on gastro-oesophageal reflux disease in ILD or came from centres that were leading the field on pathogenesis and cell therapy in ILDs. The faculty were more than willing to mingle with the participants during the breaks and I had the wonderful opportunity to discuss course matters as well as seek guidance for future possibilities. Most of the logistical issues were taken care of by two friendly ERS staff. We also had a wonderful opportunity to visit the tuberculosis museum at the Thoraxklinik itself.

The course also included an interactive multidisciplinary ILD board, and I believe more time could have been given to this and that it could have been better managed, maybe the second interactive session should have been put in earlier than at the end. Besides this the course was very well rounded and comprehensive.

Overall, the course gave me new insights into the exciting yet challenging world of ILDs. I would also like to thank the ERS on the behalf of the different forms of bursary recipients, as without the generous support from the ERS we would not have been able to attend the course. I am confident I will be able to translate the knowledge gained into clinical practice and disseminate it to the best of my abilities. These specialised training courses bring about at first an individual change and hopefully a larger change as a result. Since the mission of ERS is to promote lung health in order to alleviate suffering from disease and drive standards for respiratory medicine globally, hopefully this training adds to that mission.

\section{Conflict of interest}

Disclosures can be found alongside this article at breathe.ersjournals.com 